\title{
Epidemiology and demography of recently diagnosed cases of posterior urethral valves
}

\author{
Devesh Thakkar', Anirudh V. Deshpande ${ }^{2,3}$ and Sean E. Kennedy ${ }^{1,4,5}$
}

BACKGROUND: Posterior urethral valves (PUV) are a common cause of chronic kidney disease in young children, yet there is a paucity of knowledge about etiology and incidence. We sought to determine the incidence in Australia and to explore whether any demographic features were associated with incidence.

METHODS: PUV cases born between 2004 and 2009 in New South Wales (NSW) were identified by linkage analysis of two registries. The incidence rate was compared to registries from two other states. An audit was then performed at two pediatric centers.

RESULTS: Seventy-one boys were born and diagnosed in NSW with PUV, giving a live-birth incidence of 1.28 per 10,000. Fifty-one cases were treated at the two participating centers. Fifty-three percent of these were suspected on antenatal ultrasound. Of the remaining cases, $45 \%$ were detected in the neonatal period, with $50 \%$ of all postnatal cases presenting with urinary tract infection. No association was observed between maternal factors or socioeconomic status and PUV incidence.

CONCLUSION: PUV was found in 1 in 7,800, a similar rate to international studies. Although almost half of cases are antenatally detected, a significant proportion present outside the neonatal period. Birth incidence was not associated with maternal or demographic factors.

$\mathbf{P}$ osterior urethral valves (PUV) is a congenital malformation of the male urethra occurring early in gestational life. PUV is the most common cause of congenital bladder outlet obstruction and is responsible for $17 \%$ of pediatric end-stage kidney disease (1-3). With appropriate management, longterm complications can be prevented or minimized in some patients; nevertheless, a large number of patients suffer ongoing bladder dysfunction and progressive kidney injury, resulting in between 22 and $50 \%$ proceeding to kidney failure by early adulthood $(2,4)$.

Most reports estimate the incidence of PUV to be in the range of 1 in 5,000 to 1 in 8,000 live births. However, estimates have varied as widely as 1 in 2,000 and 1 in 25,000.
Epidemiological studies have been varied in their methodology and have generally focused on diagnostic rates rather than true incidence $(5,6)$. Two of the largest studies have been conducted in regional England, reporting on incidence/prevalence of Lower Urinary Tract Obstruction (LUTO), the majority of which are due to PUV $(5,7)$. Anumba et al. examined cases reported to a Northern Congenital Abnormality Survey between 1984 and 1997 and estimated the incidence of PUV at 1.4 per 10,000 births $(1$ in 7,100) (5). A recent retrospective study with similar methods, using data collected over 12 $\mathrm{y}$ in the Western Midlands region found 179 cases of PUV in 851,419 births (63\% of all LUTO cases), including all registered births, terminations of pregnancy and spontaneous fetal losses (7). The birth prevalence of PUV was therefore 2.1/10,000 (SD $=1.79-2.41)$. This latter study found an association between LUTO and lower socioeconomic status and ethnic background with prevalence rates in non-British European mothers being more than double rates in British European mothers. However, the study did not independently test these two factors and nonBritish European ethnicity in this region is associated with lower socioeconomic status (SES). Furthermore, the study did not separate PUV from other causes of LUTO in this analysis. Hence, it remains unclear whether deprivation and ethnicity are independently associated with PUV.

Antenatal detection of PUV improves the ability to manage the disease, allowing prenatal counseling, prompt perinatal care and organized clinical follow-up. The typical antenatal presentation of PUV is bilateral hydroureteronephrosis, with enlarged bladder on fetal ultrasound (2). Ultrasound has a high reported sensitivity of $94-95 \%$, although the specificity varies $(43-80 \%)(8,9)$.

The aims of this two-part study were firstly to determine the incidence of PUV in an Australian population and further, to test the hypothesis proposed by Malin et al. (7), that socioeconomic factors were associated with the occurrence of PUV.

\section{RESULTS}

The incidence of PUV in New South Wales (NSW) was ascertained by searching for cases reported to two databases. The

'School of Women's and Children's Health, Medicine UNSW, University of New South Wales, Sydney, New South Wales, Australia; ${ }^{2}$ Urology, Sydney Children's Hospital Network, Sydney, New South Wales, Australia; ${ }^{3}$ Centre for Kidney Research, The Children's Hospital at Westmead, Westmead, New South Wales, Australia; ${ }^{4}$ Nephrology, Sydney Children's Hospital, Randwick, New South Wales, Australia; ${ }^{5}$ Australian Centre for Perinatal Science, University of New South Wales, Sydney, New South Wales, Australia. Correspondence: Sean E. Kennedy (sean.kennedy@unsw.edu.au) 
Registry of Congenital Conditions (RoCC) records indicated 57 cases of PUV born in NSW between January 2004 and December 2009. Two of these resulted in stillbirths, and one was a termination. A further two were recorded as neonatal deaths ( $n=52$ surviving with PUV). Search of the Admitted Patient Data Collection (APDC) identified 65 infants and children born between 2004 and 2009 who were hospitalized with PUV in the July 2003-June 2010 period. Of these, 46 were registered on the RoCC, with 19 having no linkage. The combined registry data was used as the numerator $(52+19$ $=71)$ to provide the estimate of incidence for PUV. In total, 554,289 live births were recorded in NSW between 2004 and 2009. This provides a live-birth incidence estimate of 1.28 per 10,000 live births.

To explore whether the birth incidence in NSW was representative of other states in Australia, we made inquiries to data collections in two other states. The reporting periods varied between states. The Western Australian Register of Developmental Anomalies, which records congenital anomalies diagnosed up to $6 \mathrm{y}$ of age, indicated 18 cases of PUV in Western Australia during the 2004-2009 calendar years inclusive. There were 171,897 live births in Western Australia during the same period, giving a live-birth incidence of 1.05 per 10,000. The South Australian Birth Defects Register records anomalies detected before $5 \mathrm{y}$ of age and recognized 14 cases of PUV between 2003 and 2007, with 91,466 recorded live births in South Australia in the same period. The live-birth incidence in South Australia was thus estimated at 1.53 per 10,000 live births.

We next conducted a more detailed analysis of the cases of PUV that had been treated in the two largest children's hospitals in NSW. A total of 51 boys born between 2004 and 2009 were treated for PUV at the two hospitals in the Sydney Children's Hospitals Network. That is, 72\% (51/71) of all cases identified in the state of NSW during that period. The other 20 cases must have been managed in other hospitals not included in this study. Two cases in which the child was born outside Australia were excluded. The cohort consisted of 50 singleton pregnancies and 1 twin pregnancy; in the latter case, the other child (a male) was unaffected by PUV. During 2004 and 2010, only three cases of PUV were diagnosed in boys aged more than $5 \mathrm{y}$.

To test whether the incidence of PUV was higher in areas of less socioeconomic advantage, we grouped families according to postal codes. Postcode data was matched to quintiles for each case $(1=$ lowest quintile, $5=$ highest quintile). The majority of families were in the upper two quintiles, with only four cases in the lowest quintile. The overall mean socioeconomic quintile for our cohort was 3.8. Quintiles are standardized in each state, and so the overall mean in NSW was 3.0. No child with PUV was identified as of Indigenous origin. All parents spoke English.

The diagnosis of PUV was suspected after antenatal ultrasonography in 27 cases (53\%). Gestational age at time of detection was documented in 12 cases, with a median of $23.5 \mathrm{wk}$ (inter-quartile range; IQR $=18.5-34.8$ ). Ten of 24 cases that were not diagnosed after antenatal screening were diagnosed before 1 mo of age. Presentation with a UTI accounted for half of these. The remaining presentations were variable. For those detected outside the neonatal period, the median age of presentation was $4 \mathrm{mo}(\mathrm{IQR}=3-6)$.

Due to the method of documentation, much pregnancy related and perinatal data was available only for perinatally treated PUV. In the cases for which maternal age was documented $(n=26)$, the median maternal age of the cohort was $30.5 \mathrm{y}(\mathrm{IQR}=22.5-35.3)$ as compared to $30.0 \mathrm{y}$ in the general Australian population in 2006. Fifty-two percent (12/23) of mothers who had parity documented were first time mothers (national rate $=41 \%, P=0.21$ ). Eight mothers (35\%) had given birth only once previously. One mother developed gestational diabetes during the pregnancy, while another developed hypertensive disease of pregnancy. Three mothers were smokers before and throughout the pregnancy, with one of these additionally suffering from asthma. One mother had been diagnosed with hypothyroidism prior to the pregnancy.

The mean length of gestation documented in this cohort was $37.3 \pm 2.0 \mathrm{wk}(n=26)$. This figure is slightly lower than the national average in 2006 of $38.8 \mathrm{wk}$. Eighteen of $26(69 \%)$ babies were born full term ( $37 \mathrm{wk}$ or greater). This is significantly lower than the national rate of $90.6 \%$ in $2006(P<0.001)$. All preterm births were 33-36 wk gestation.

Birth weight data was recorded for 25 cases, with a mean of $3,156 \pm 610 \mathrm{~g}$. The national average birth weight is $3,370 \mathrm{~g}$. Twelve percent $(3 / 25)$ of patients were low birth weight $(<2,500 \mathrm{~g})$, compared to a national average of $6.4 \%(P=0.21)$. No child was classified as very low birth weight $(<1,500 \mathrm{~g})$ or extremely low birth weight $(<1,000 \mathrm{~g})$. Vaginal deliveries occurred in 13 cases, with two being assisted vaginal deliveries. Seven babies were delivered by Caesarean, giving a Caesarean rate of $35 \%$. The national rate of Caesarean was $31 \%$ in $2006(P=0.68)$. Documentation of amniotic fluid volumes was poor, but oligohydramnios was noted in nine cases, one of which was anhydramniotic. All of these also had antenatally detected PUV.

One neonate was found to have Trisomy 21, while another had congenital pulmonary valve stenosis. One of the boys who was diagnosed with PUV after the age of $5 \mathrm{y}$ (at $8 \mathrm{y}$ of age) also had Trisomy 21. A family history of kidney disease was noted in only one child, whose father had vesicoureteric reflux and a nonfunctioning kidney.

Micturating cystourethrogram was the major method of diagnosis, utilized in 37 cases (80.4\%). Cystoscopy was utilized in the remaining cases either because micturating cystourethrogram was unable to be performed or as a curative procedure during which valves were also ablated.

\section{DISCUSSION}

As far as we know, this study is the first PUV focused incidence and demographic study conducted outside of North America or Europe. We utilized state-wide data collection services from three distinct regions to estimate the live-birth incidence of PUV. Using combined data from the two registries in NSW, we 


\section{Articles | Thakkare tal.}

estimated a live-birth incidence of 1.28 per $10,000(1$ in 7,800). The registries from Western Australia and South Australia include cases diagnosed up to 6 and 5 y respectively. The birth incidence data provided from these states was similar to NSW and all were close to the approximation provided by the earlier large English study of 1.4 per 10,000 but less than the recent study of 2.1 per 10,000 $(5,7)$. Notably, the latter study included all pregnancies and noted that terminations of pregnancy occurred in $24.6 \%$.

Reporting to the Australian registers is mandatory by law and hospital services have various routine and automatic reporting processes; therefore, it is expected that coverage rates will be reasonably high. Two potential problems when using registries of congenital defects to estimate birth incidence are under-reporting of recognized cases and delayed diagnoses. We sought to address both of these issues by linking with an inpatient database of cases treated before the age of 5 $y$. We identified a small but significant rate of under-reporting to the congenital registry. Some of these cases may have been children born outside of NSW, as shown by the finding that two children treated at the children's hospitals in this period were born outside of Australia. Interestingly, a similar number of boys registered in the congenital register did not appear as inpatients in NSW. This could be explained by treatment in other states or territories or reclassification of diagnoses.

The incidence rate is expected to be an underestimate due to noninclusion of cases that are detected after $5 \mathrm{y}$ of life. We sought to address this by review of inpatient records at the children's hospitals. We identified only three boys who were diagnosed after the age of $5 \mathrm{y}$ ( $6 \%$ of those diagnosed before $5 \mathrm{y})$. It is possible that a further small number of cases will not present till adulthood and would not have been seen in the children's hospitals.

Our medical record audit identified more than $70 \%$ of cases reported to the NSW registries and can thus be considered to provide a broadly representative sample. Importantly, despite almost universal use of antenatal ultrasonography in Australia, $45 \%$ of cases were not suspected before delivery. Most of the remaining cases were diagnosed during the first year of life, with urinary tract infections being the presenting feature in half of them. This antenatal detection rate is similar to other reports and highlights the importance of including PUV in a list of provisional diagnoses for boys with UTI or other genitourinary symptoms. We found that $80 \%$ of cases were detected by $1 \mathrm{y}$ of age, which is an earlier average age than previously reported $(1,2)$. Schober $e$ t al. described the mean age of late presentation as $7.4 \mathrm{y}$; however, their study utilized data from as far back as 1990 , and it is expected this rate is lower presently given the more widespread use of antenatal ultrasonography (10).

Only one child with PUV was found to have a potentially significant renal family history which is consistent with the view that PUV are unlikely to be related to a single genetic defect. Similarly, only one boy in the observed set of twins was affected by PUV. However, we identified two cases of PUV in children with Trisomy 21 . One of these was born in our study period, while another was diagnosed beyond the age of $5 \mathrm{y}$.
A total of 599 stillbirths or live births with Trisomy 21 were reported to the RoCC between 2003 and 2009; therefore, this one case of PUV would give an incidence of 16.9 per 10,000 Trisomy 21 births. While this is clearly an unreliable estimate, it does support previous reports of a possible association between Trisomy 21 and PUV (11).

We did not identify any association between demographic factors and PUV. Specifically, unlike the recent English study (7), we did not find higher rates of PUV in economically disadvantaged families. Our method of allocating SES by postal code is an established methodology in Australia and is similar to that used in the UK study. This method of classifying SES is dependent on postal areas, which vary in population levels and fertility rates. Accurate SES quintiles are not available for each individual case which could lead to a degree of ecological fallacy in our results. This would need to be addressed by collection of detailed individual SES data which was beyond the scope of our study. As opposed to the findings of Malin et al., our results could potentially be interpreted to suggest that the risk of PUV births is higher in less disadvantaged groups; however, a confounding factor we were unable to control for is overall birth rate according to SES. Crude birth rates in NSW according to postcode are not available instead birth rates are reported according to Local Health Districts in the Health Statistics NSW database (NSW Government, Sydney, Australia). Crude birth rates according to Local Health Districts in 2006 ranged from 7.4 to 16.4 per 1,000 population and all families in our study who lived in Local Health Districts with birth rates less than 10 per 1,000, also lived in the lowest two quintiles for SES. Hence, the relatively low numbers of families in our study from disadvantaged areas may reflect an overall lower birth rate in those areas. Our data collection methods did not allow us to identify racial origin. We could therefore not test the finding of higher rates of PUV in children of non-European ancestry.

The retrospective nature of this study limited the availability of complete and detailed data. Specifically, much perinatal data were not documented for cases diagnosed outside the neonatal period. As these cases are typically less severe than those diagnosed in the perinatal period, it is possible that our data has been biased towards the more severe cases.

\section{Conclusion}

Our study suggests that PUV occur in 1 in every 7,000-8,000 live births. Although the majority of cases are detected in the neonatal period, a significant proportion are not detected until later and the possibility of the diagnosis should be considered in boys with UTI and/or urological symptoms. The aetiology of PUV remains unclear, we did not find any maternal factors associated with PUV incidence nor did our findings support an association with socioeconomic status.

\section{METHODS}

\section{Incidence and Reporting Rates}

We ascertained the number of cases of PUV reported to three different state-based registries in Australia. The RoCC collects data of scheduled congenital conditions in New South Wales (NSW). 
Reporting occurs at a hospital level, with clinicians required to submit a prescribed form for each case identified in utero or within the first year of life. Data are coded and stored by calendar year. The number of reported PUV cases from beginning 2004 till end of 2009 were obtained using the unique code "75360". Further data was obtained from the Western Australian Register of Developmental Anomalies which has similar reporting guidelines but includes cases identified before the age of $6 y$, and the South Australian Birth Defects Register which includes cases diagnosed before $5 \mathrm{y}$ of age.

A linkage analysis was performed linking data from the RoCC with the NSW APDC. The APDC stores data on all hospital admissions in NSW, such as patient demographics and clinical information linked to Australian defined diagnosis related groups. Patients who had been hospitalized with PUV were identified by the code "Q64.21" for any diagnosis with PUV, and the code used for "congenital posterior urethral, unspecified" "Q64.20." Linkage of NSW datasets was carried out by the Centre for Health Record Linkage $(\mathrm{CHeReL})$ with the approval of the NSW Population and Health Services Research Ethics Committee; analysis of de-identified linked data was carried out by the Centre for Epidemiology and Evidence in the NSW Ministry of Health and aggregate data was provided to the investigators. APDC data were provided in two sets as number of distinct patients born and admitted with diagnosis PUV under $1 \mathrm{y}$ of age and under $5 \mathrm{y}$ of age respectively in the 2004-2009 period.

The combined data from the RoCC and APDC was used to approximate the incidence of PUV in NSW in conjunction with total live-birth rate in NSW provided by the Perinatal and Reproductive Epidemiology Research Unit. This figure thus pertains only to completed live births and will exclude terminations, miscarriage, and still births.

\section{Demographics and Detection}

We next performed a retrospective study of cases of PUV managed at the two largest tertiary children's hospitals in NSW between 1 January 2004 and 31 December 2009. Cases were identified by search of medical records and departmental databases. The medical records search utilized the diagnosis related group for "Posterior Urethral Valves" (code Q64.21) and therefore would have identified cases that were also reported to the ADPC. We also searched using the diagnosis related group "Endoscopic destruction of urethral valve" (code 37854-00).

Case notes were reviewed to obtain demographic details, maternal history, pregnancy details, neonatal details including birth weight and gestational age, timing, and mode of diagnosis. A diagnosis of PUV was confirmed by either cystoscopic or radiologic findings. Radiologic definition was a significantly dilated posterior urethra causing obstruction to antegrade flow, usually associated with changes in the bladder and upper tracts. At cystoscopy, PUV were diagnosed by the presence of valve leaflets attached immediately distal to the veru montanum or rarely as a circumferential membrane with a central aperture. Cases with urethral atresia and anterior urethral pathologies were excluded.

Population-based normative data were provided by Perinatal and Reproductive Epidemiology Research Unit. We further divided birth weight and gestational age into low birth weight (birth weight $<2,500 \mathrm{~g}$ ) and preterm (length of gestation $<37 \mathrm{wk}$ ) categories.

Socioeconomic status was determined using postal codes of place of residence at time of first presentation, using the Socio-economic Indexes for Areas, 2006, Index of Relative Socio-economic Advantage and Disadvantage (12). This data is determined from the five yearly national census; we chose to use 2006 data as representative of the birth cohort between 2004 and 2010. The cohort was divided into quintiles by postcode. Race or ethnicity was not reliably coded in the case notes except for Indigenous status. Language spoken at home was recorded.

Ethical approval for the review of cases in two hospitals was provided by the Human Research Ethics Committee of the Sydney Children's Hospital Network.

\section{Statistical Analyses}

Incidence values were calculated using combined data from 20032009 available from Perinatal and Reproductive Epidemiology Research Unit as required (13) (http://npesu.unsw.edu.au; accessed October 2013); total live-birth figures (both male and female) were used. For other analyses, we used 2006 rates and figures for the general population.

Nonparametric data is reported as median (IQR). Means were calculated for length of gestation and birth weight, reported as mean \pm SD.

$\chi^{2}$ tests were used to establish significance as compared to these general population figures.

\section{ACKNOWLEDGMENTS}

Lee Taylor and Helen Moore, Centre for Epidemiology and Research, NSW Ministry of Health; Carol Bower, Western Australian Register of Developmental Anomalies; and Heather Scott, SA Birth Defects Register. Robert Farnsworth, Sydney Children's Hospital, for allowing access to his personal case files.

Disclosure: The authors declare no conflict of interest.

\section{REFERENCES}

1. Agarwal S. Urethral valves. BJU Int 1999;84:570-8.

2. Hodges SJ, Patel B, McLorie G, Atala A. Posterior urethral valves. ScientificWorldJournal 2009;9:1119-26.

3. Krishnan A, de Souza A, Konijeti R, Baskin LS. The anatomy and embryology of posterior urethral valves. J Urol 2006;175:1214-20.

4. Heikkilä J, Holmberg C, Kyllönen L, Rintala R, Taskinen S. Long-term risk of end stage renal disease in patients with posterior urethral valves. J Urol 2011;186:2392-6.

5. Anumba DO, Scott JE, Plant ND, Robson SC. Diagnosis and outcome of fetal lower urinary tract obstruction in the northern region of England. Prenat Diagn 2005;25:7-13.

6. Dinneen MD, Duffy PG. Posterior urethral valves. Br J Urol 1996;78: $275-81$.

7. Malin G, Tonks AM, Morris RK, Gardosi J, Kilby MD. Congenital lower urinary tract obstruction: a population-based epidemiological study. BJOG 2012;119:1455-64.

8. Lissauer D, Morris RK, Kilby MD. Fetal lower urinary tract obstruction. Semin Fetal Neonatal Med 2007;12:464-70.

9. Bernardes LS, Aksnes G, Saada J, et al. Keyhole sign: how specific is it for the diagnosis of posterior urethral valves? Ultrasound Obstet Gynecol 2009;34:419-23.

10. Schober JM, Dulabon LM, Woodhouse CR. Outcome of valve ablation in late-presenting posterior urethral valves. BJU Int 2004;94:616-9.

11. Kupferman JC, Druschel CM, Kupchik GS. Increased prevalence of renal and urinary tract anomalies in children with Down syndrome. Pediatrics 2009;124:e615-21.

12. Australian Bureau of Statistics. Socio-economic Indexes for Areas, 2006. (http://www.abs.gov.au/websitedbs/censushome.nsf/home/seifa.)

13. National Perinatal Epidemiology and Statistics Unit. Australia’s mothers and babies, 2012. (http://www.npesu.unsw.edu.au/surveillance/australia\% E2\%80\%99s-mothers-and-babies-2011\#prev reports.) 